\title{
Riffle shuffles of a deck with repeated cards
}

\author{
Sami Assaf $\|$ Persi Diaconis $\|$ and K. Soundararajan $\|^{3}$ \\ ${ }^{1}$ Department of Mathematics, Massachusetts Institute of Technology, 77 Massachusetts Avenue, Cambridge, MA \\ 02139-4307 \\ ${ }^{2}$ Department of Statistics, Stanford University, 390 Serra Mall Stanford, CA 94305-4065 \\ ${ }^{3}$ Department of Mathematics, Stanford University, 450 Serra Mall, Building 380, Stanford, CA 94305-2125
}

\begin{abstract}
We study the Gilbert-Shannon-Reeds model for riffle shuffles and ask 'How many times must a deck of cards be shuffled for the deck to be in close to random order?'. In 1992, Bayer and Diaconis gave a solution which gives exact and asymptotic results for all decks of practical interest, e.g. a deck of 52 cards. But what if one only cares about the colors of the cards or disregards the suits focusing solely on the ranks? More generally, how does the rate of convergence of a Markov chain change if we are interested in only certain features? Our exploration of this problem takes us through random walks on groups and their cosets, discovering along the way exact formulas leading to interesting combinatorics, an 'amazing matrix', and new analytic methods which produce a completely general asymptotic solution that is remarkable accurate.
\end{abstract}

Keywords: card shuffling, lumping of Markov chains, Poisson summation

\section{Introduction}

A basic question in scientific computing is 'How many times must an iterative procedure be run?'. A basic answer is 'It depends.'. In this paper we study the mixing properties of the Gilbert-Shannon-Reeds model [19, 21] for riffle shuffling a deck of $n$ cards and ask how many times the deck must be shuffled for the cards to be in close to random order. Our answer depends not only on the metric we use to measure distance to uniformity, but also on the particular properties of the deck that are of interest.

To be precise, we consider a 'deck' to be a multiset of $n$ cards. We shuffle the deck by first cutting it into two piles according to the binomial distribution, and then riffling the piles together by successively dropping cards from either pile with probability proportional to the size. This process defines a measure, denoted $Q_{2}(\sigma)$, on the symmetric group $\mathcal{S}_{n}$. Repeated shuffles are defined by convolution powers

$$
Q_{2}^{* k}(\sigma)=\sum_{\omega \cdot \tau=\sigma} Q_{2}(\tau) Q_{2}^{*(k-1)}(\omega) .
$$

\footnotetext{
${ }^{\dagger}$ Research supported by MSRI Postdoctoral Research Fellowship

${ }^{\ddagger}$ Research supported by NSF grant DMS-06-03886 and the CNRS chair d'excellence at the University of Nice, Sophia-Antipolis

$\S$ Research supported by NSF grant DMS 0500711 
This shuffling model, which accurately models how most people actually shuffle a deck of cards, was introduced by Gilbert and Shannon [19] and independently by Reeds [21].

Bayer and Diaconis [3] generalized this to $a$-shuffles, which is the natural extension to shuffling with $a$ hands: the deck is cut into $a$ packets by multinomial distribution and cards are successively dropped from packets with probability proportional to packet size. Letting $Q_{a}(\sigma)$ denote this measure, they show that convolution of general $a$-shuffles is as nice as possible, namely

$$
Q_{a} * Q_{b}=Q_{a b} .
$$

Thus it is enough to study a single $a$-shuffle of the deck.

To that end, denote the uniform distribution by $U=U(\sigma)$. For a deck with $n$ distinct cards, $U=1 / n$ !, and for a more general deck with $D_{1} 1$ 's, $D_{2} 2$ 's, up to $D_{m} m$ 's, we have $U=1 /\left(\begin{array}{c}D_{1}+\cdots+D_{m} \\ D_{1}, \ldots, D_{m}\end{array}\right)$. There are several ways to measure the distance between $Q_{a}$ and $U$, though for the purposes of this paper we restrict our attention to total variation distance and separation distance.

The total variation distance is defined by

$$
\left\|Q_{a}-U\right\|_{T V}=\max _{\text {subsets } A}\left|Q_{a}(A)-U(A)\right|=\frac{1}{2} \sum_{\sigma}\left|Q_{a}(\sigma)-U(\sigma)\right| .
$$

In general, the formulas for $Q_{a}(\sigma)$ may be quite complicated, making calculations of total variation intractable. Therefore we will also consider the separation distance defined by

$$
\operatorname{SEP}(a)=\max _{\sigma} 1-\frac{Q_{a}(\sigma)}{U(\sigma)} .
$$

Here, only a single probability needs to be computed, though as we shall see even that can be difficult. From the formulas above, one can easily see that separation provides an upper bound for total variation, which makes separation a good measure to use when total variation becomes too complicated to compute.

In widely cited works, Aldous [2] and Bayer and Diaconis [3] show that $\frac{3}{2} \log _{2}(n)+c$ shuffles are necessary and sufficient to make the total variation distance small, while $2 \log _{2}(n)+c$ shuffles are necessary and sufficient to make separation small. These results, however, look at all aspects of a permutation, i.e. consider a deck with distinct cards. In many card games, only certain aspects of the permutation matter. For instance, in Baccarat, suits are irrelevant and all 10's and picture cards are equivalent, and in ESP card guessing experiments, a Zener deck of 25 cards with each of 5 symbols repeated five times is used. It is natural, therefore, to ask how many shuffles are required in these situations, and so we consider a deck to have repeated cards.

Many results are known for how long it takes certain features of a permutation, e.g. longest cycle, descent structure, etc, to become random; for a thorough treatment of such results see [11]. The particular problem we address in this paper was first addressed by Conger and Viswanath [8, 9] who derive remarkable numerical procedures giving useful answers for cases of practical interest.

In this paper, we present many of our main results from [?], giving exact formulae and asymptotics for a deck of $n$ cards with $D_{1}$ cards labelled $1, D_{2}$ cards labelled $2, \ldots, D_{m}$ cards labelled $m$. Our results are proved from the deck starting 'in order', i.e. with 1's on top through $m$ 's at the bottom. In Section 2. we show that the processes we study are Markov by framing the problem in the context of random walks on cosets. We derive a formula for the transition matrix following a single card in Section 3 , and show 
that this matrix shares many properties with Holte's 'Amazing Matrix' [20]. In Section 4 we consider a general deck, limiting our metric to the separation distance, and derive new formulae and asymptotic approximations which we unify into our 'rule of thumb' formula. Section 5 shows that our results depend on the initial configuration of the deck, a fact also observed by Conger and Viswanath [8, 9, ?]. This extended abstract contains precise statements of our main results along with the main ideas of the proofs; for full details see [?].

\section{Random walks on Young subgroups}

In this section, we reformulate shuffling in terms of random walks on a finite group, so that our investigation of particular properties of a deck becomes a quotient walk on Young subgroups of $\mathcal{S}_{n}$.

Let $G$ be a finite group, and let $Q$ be a probability on $G$, i.e. $Q(g) \geq 0$ and $\sum_{g \in G} Q(g)=1$. Take a random walk on $G$ by repeatedly choosing elements independently from $G$ with probability $Q$, say $g_{1}, g_{2}, g_{3}, \ldots$, and, beginning with the identity element $1_{G}$, multiply on the left by $g_{i}$. This generates the following sequence of elements, the left walk,

$$
1_{G}, g_{1}, g_{2} g_{1}, g_{3} g_{2} g_{1}, \ldots
$$

By inspection, the chance that the walk is at $g$ after $k$ steps is given by convolution formula $11 Q^{* k}(g)$, where $Q^{0}(g)=\delta_{1_{G}, g}$.

To focus on certain aspects of the walk, we choose a subgroup and consider the quotient walk as follows. Let $H \leq G$ be a subgroup of $G$, and let $X$ denote the set of left cosets of $H$ in $G$, i.e. $X=G / H=\{x H\}$. The quotient walk on $X$ is derived from the left walk on $G$ by reporting the coset to which the current position of the walk belongs. This defines a Markov chain on $X$ with transition matrix given by

$$
K(x, y)=Q\left(y H x^{-1}\right)=\sum_{h \in H} Q\left(y h x^{-1}\right) .
$$

Note that $K$ is well-defined (i.e. independent of the choice of coset representatives) and doubly stochastic. Thus the uniform distribution on $X, U=|H| /|G|$, is a stationary distribution for $K$. The following result, showing that powers of $K$ correspond precisely to convolving and taking cosets, is intuitively obvious with a straightforward proof.

Proposition 2.1 For $Q$ a probability distribution on a finite group $G$ and $K$ as defined in (5), we have

$$
K^{l}(x, y)=Q^{* l}\left(y H x^{-1}\right) .
$$

We may identify permutations in $\mathcal{S}_{n}$ with arrangements of a deck of $n$ cards by setting $\sigma(i)$ to be the label of the card at position $i$ from the top. For instance, the permutation 2143 is associated with four cards where " 2 " is on top, followed by " 1 ", followed by " 4 ", and finally " 3 " is on the bottom. Therefore the random walk on $\mathcal{S}_{n}$ with probability $Q_{2}$ corresponds precisely to riffle shuffles of a deck of $n$ distinct cards. If we consider the first $D_{1}$ cards to be labelled "1", the next $D_{2}$ cards to be labelled "2", and so on up to the last $D_{m}$ cards labelled " $m$ ", then this corresponds precisely to the coset space of a Young subgroup,

$$
X=\mathcal{S}_{n} /\left(\mathcal{S}_{D_{1}} \times \mathcal{S}_{D_{2}} \times \cdots \times \mathcal{S}_{D_{m}}\right) .
$$

Thus Proposition 2.1 shows that the processes studied in the body of this paper are Markov chains. 


\section{A new 'amazing' matrix}

Suppose the ace of spades is on the bottom of a deck of $n$ cards. How many shuffles does it take until this one card is close to uniformly distributed on $\{1,2, \ldots, n\}$ ? We analyze this problem by writing down the transition matrix following a single card through an otherwise indistinguishable deck.

Proposition 3.1 Let $P_{a}(i, j)$ be the chance that the card at position $i$ moves to position $j$ after an ashuffle. For $1 \leq i, j \leq n, P_{a}(i, j)$ is given by

$$
\frac{1}{a^{n}} \sum_{k=1}^{a} \sum_{r=l}^{u}\left(\begin{array}{c}
j-1 \\
r
\end{array}\right)\left(\begin{array}{c}
n-j \\
i-r-1
\end{array}\right) k^{r}(a-k)^{j-1-r}(k-1)^{i-1-r}(a-k+1)^{(n-j)-(i-r-1)}
$$

where $r$ ranges from $l=\max (0,(i+j)-(n+1))$ to $u=\min (i-1, j-1)$.

Proof: Consider the number of ways that an inverse $a$-shuffle can bring the card at position $j$ to position $i$. First, the card at position $j$ must have come from some pile, say $k, 1 \leq k \leq a$. Say $r$ of the cards above this came from piles 1 to $k$, and so the remaining $j-1-r$ came from piles $k+1$ to $a$. Those $r$ cards all must appear before the card at position $j$ in $\left(\begin{array}{c}j-1 \\ r\end{array}\right)$ ways. This leaves $i-1-r$ cards below position $j$ which came from piles 1 to $k-1$ in $\left(\begin{array}{c}n-j \\ i-r-1\end{array}\right)$ ways, and the remaining cards must be from piles $k$ to $a$.

For example, the $n \times n$ transition matrices for $n=2,3$ are given below.

$$
\frac{1}{2 a}\left(\begin{array}{cc}
a+1 & a-1 \\
a-1 & a+1
\end{array}\right) \quad \frac{1}{6 a^{2}}\left(\begin{array}{ccc}
(a+1)(2 a+1) & 2\left(a^{2}-1\right) & (a-1)(2 a-1) \\
2\left(a^{2}-1\right) & 2\left(a^{2}+2\right) & 2\left(a^{2}-1\right) \\
(a-1)(2 a-1) & 2\left(a^{2}-1\right) & (a+1)(2 a+1)
\end{array}\right)
$$

These matrices share many properties, given in Proposition 3.2. with the 'amazing matrix' discovered by Holte [20] in his study of the 'carries process' of ordinary addition. Diaconis and Fulman [12] show that Holte's matrix is also the transition matrix for the number of descents in repeated $a$-shuffles. We have not been able to find a closer connection between the two matrices.

Proposition 3.2 The transition matrices following a single card have the following properties:

1. they are cross-symmetric, i.e. $P_{a}(i, j)=P_{a}(n-i+1, n-j+1)$;

2. they are multiplicative, i.e. $P_{a} \cdot P_{b}=P_{a b}$;

3. the eigenvalues form the geometric series $1,1 / a, 1 / a^{2}, \ldots, 1 / a^{n-1}$;

4. the right eigen vectors are independent of $a$ and have the simple form: $V_{m}(i)=(i-1)^{i-1}\left(\begin{array}{c}m-1 \\ i-1\end{array}\right)+(-1)^{n-i+m}\left(\begin{array}{c}m-1 \\ n-i\end{array}\right)$ for $1 / a^{m}, m \geq 1$.

Proof: The cross-symmetry (1) follows from Proposition 3.1. and the multiplicative property (2) follows from the shuffling interpretation and equation (2). Property (1) implies that the eigen structure is quite constrained. Properties (3) and (4) follow from results of Cuicu [7].

The following Corollary also follows as a special case of Theorem 2.2 in [8]. 
Corollary 3.3 Consider a deck of $n$ cards with the ace of spades starting at the bottom. The chance that the ace of spades is at position $j$ from the top after an a-shuffle is

$$
Q_{a}(j)=P_{a}(n, j)=\frac{1}{a^{n}} \sum_{k=1}^{a}(k-1)^{n-j} k^{j-1} .
$$

From the explicit formula, we are able to give exact numerical calculations and sharp asymptotics for any of the distances to uniformity. The results below show that $\log _{2} n+c$ shuffles are necessary and sufficient for both separation and total variation (and there is a cutoff for these). This is surprising since, on the full permutation group, separation requires $2 \log _{2} n+c$ steps whereas total variation requires $\frac{3}{2} \log _{2} n+c$. Of course, for any specific $n$, these asymptotic results are just indicative.

Tab. 1: Distance to uniformity for a deck of 52 cards. The upper table assumes distinct cards, and the lower table follows a single card starting at the bottom of the deck.

\begin{tabular}{c|cccccccccccc}
\hline & 1 & 2 & 3 & 4 & 5 & 6 & 7 & 8 & 9 & 10 & 11 & 12 \\
\hline$T V$ & 1.00 & 1.00 & 1.00 & 1.00 & .924 & .614 & .334 & .167 & .085 & .043 & .021 & .010 \\
SEP & 1.00 & 1.00 & 1.00 & 1.00 & 1.00 & 1.00 & 1.00 & .996 & .931 & .732 & .479 & .278 \\
\hline \multicolumn{1}{c}{} & 1 & 2 & 3 & 4 & 5 & 6 & 7 & 8 & 9 & 10 & 11 & 12 \\
\hline$T V$ & .873 & .752 & .577 & .367 & .200 & .103 & .052 & .026 & .013 & .007 & .003 & .002 \\
SEP & 1.00 & 1.00 & .993 & .875 & .605 & .353 & .190 & .098 & .050 & .025 & .013 & .006 \\
\hline
\end{tabular}

Remarks on Table 1 We use Proposition 3.1 to give exact results when $n=52$. For comparison, the upper table gives exact results for the full deck using [3]. The lower table shows that it takes about half as many shuffles to achieve a given degree of mixing for a card at the bottom of the deck. For example, the widely cited ' 7 shuffles' for total variation drops this distance to .334 for the full ordering, but this requires only 4 shuffles to achieve a similar degree of randomness for a single card at the bottom.

For asymptotic results, we first derive an approximation to separation, which also serves as an upper bound for total variation. Finally, we derive a matching lower bound for total variation. Proofs have been omitted for brevity, but again full details are available in [?].

Proposition 3.4 After an a-shuffle, the probability that the bottom card is at position $i$ satisfies

$$
\frac{1}{a} \frac{\alpha^{n-i+1}}{1-\alpha^{n}} \leq Q_{a}(i) \leq \frac{1}{a} \frac{\alpha^{n-i}}{1-\alpha^{n-1}},
$$

where for brevity we have set $\alpha=1-1 / a$. In particular, the separation distance satisfies

$$
1-\frac{n}{a} \frac{\alpha^{n}}{1-\alpha^{n}} \leq \operatorname{SEP}(a) \leq 1-\frac{n}{a} \frac{\alpha^{n-1}}{1-\alpha^{n-1}} .
$$


If $a=2^{\log _{2}(n)+c}=n 2^{c}$, then our result shows that the $\operatorname{SEP}(a)$ is approximately

$$
1-\frac{1}{2^{c}} \frac{e^{-2^{-c}}}{1-e^{-2^{-c}}}
$$

and for large $c$ this is $\approx 2^{-c-1}$. The fit to the data in Table 1 is excellent: for example after ten shuffles of a fifty-two card deck we have $2^{-c-1}=\frac{26}{1024}$ which is very nearly the observed separation distance of 0.025 .

Remark 3.5 Proposition 3.4 gives a local limit for the probability that the original bottom card is at position $j$ from the bottom. When the number of shuffles is $\log _{2} n+c$, the density of this (with respect to the uniform measure) is asymptotically $z(c) e^{-j / 2^{c}}$, with $z$ a normalizing constant $\left(z(c)=1 / 2^{c}\left(e^{j / 2^{c}}-1\right)\right)$. The result is uniform in $j$ for $c$ fixed, $n$ large.

Proposition 3.6 Consider a deck of $n$ cards with the ace of spades at the bottom. With $\alpha=1-1 / a$, the total variation distance for the mixing of the ace of spades after an a-shuffle is at most

$$
\frac{\alpha^{n+1}}{1-\alpha^{n}}-\frac{a \alpha^{2}\left(1-\alpha^{n-1}\right)}{n\left(1-\alpha^{n}\right)}+\frac{1}{n \log (1 / \alpha)} \log \left(\frac{a}{n} \frac{1-\alpha^{n}}{\alpha^{n+1}}\right)
$$

and at least

$$
\frac{\alpha^{n}}{1-\alpha^{n-1}}-\frac{a\left(1-\alpha^{n}\right)}{n \alpha\left(1-\alpha^{n-1}\right)}+\frac{1}{n \log (1 / \alpha)} \log \left(\frac{a}{n} \frac{1-\alpha^{n-1}}{\alpha^{n-1}}\right) .
$$

After $\log _{2} n+c$ shuffles, that is when $a=2^{c} n$, Proposition 3.6 shows that the total variation distance is approximately (with $C=2^{c}$ )

$$
C \log \left(C\left(e^{1 / C}-1\right)\right)+\frac{1-C \log \left(e^{1 / C}-1\right)}{\left(e^{1 / C}-1\right)} .
$$

Thus when $c$ is 'large and negative,' the total variation is close to 1 , and when $c$ is large and positive, the total variation is close to 0 . Thus total variation and separation converge at the same rate. This is an asymptotic result and, for example, Table 1 supports this.

Similar, but more demanding, calculations show that if the ace of spades starts at position $i$, and $\max (i / n,(n-i) / n) \geq A>0$ for some fixed positive $A$, then $\frac{1}{2} \log _{2} n$ shuffles suffice for convergence in any of the metrics. We omit further details.

\section{Separation distance for the general case}

A main result of Bayer and Diaconis [3] is the simple formula for an $a$-shuffle of a deck of $n$ distinct cards:

$$
Q_{a}(\sigma)=\frac{1}{a^{n}}\left(\begin{array}{c}
n+a-r \\
n
\end{array}\right),
$$

where $r=r(\sigma)$ is the number of rising sequences in $\sigma$, equivalently one more than the number of descents in $\sigma^{-1}$. This formula allows simple closed form expressions for a variety of distances as well as asymptotic analysis. 
In this section we work with general decks containing $D_{i}$ cards labelled $i, 1 \leq i \leq m$. The formulae of this section hardly resemble the elegant expression above. Further, we only give precise formula for the least likely deck. The following lemma shows that this deck, where the separation distance is achieved, is the reverse the initial deck configuration. This is equivalent to Theorem 2.1 from [8].

Proposition 4.1 Let $D$ be a deck as above. After an a-shuffle of the deck with 1 's on top down to $m$ 's on bottom, the least likely configuration is the reverse deck $w^{*}$ with $m$ 's on top down to 1 's on the bottom.

Proof: The only cuts of the initial deck resulting in $w^{*}$ are those containing no pile with distinct letters. For all such cuts, each rearrangement of the deck is equally likely to occur.

While finding a completely general formula for $Q_{a}(w)$ for arbitrary $w$ is infeasible, below we do this for $w^{*}$.

Theorem 4.2 Consider a deck with $n$ cards and $D_{i}$ cards labeled $i, i=1, \ldots, m$. Then the separation distance after an a-shuffle of the sorted deck ( 1 's followed by 2 's, etc) is given by

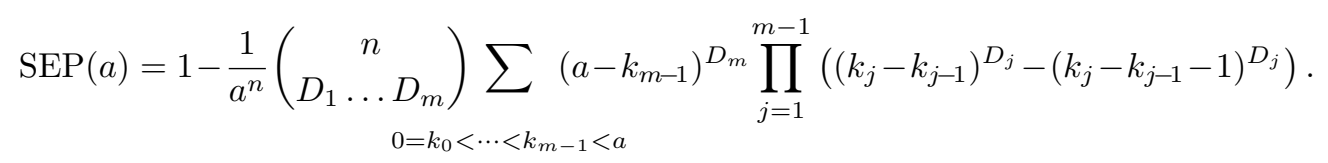

Proof: From the analysis in the proof of Proposition $4.1, Q_{a}\left(w^{*}\right)$ is given by

$$
Q_{a}\left(w^{*}\right)=\sum_{\substack{A_{1}+\cdots+A_{a}=n \\
A \text { refines } D}} \frac{1}{a^{n}}\left(\begin{array}{c}
n \\
A_{1}, \ldots, A_{a}
\end{array}\right) \frac{1}{\left(\begin{array}{c}
n \\
D_{1}, \ldots, D_{m}
\end{array}\right)},
$$

where ' $A$ refines $D$ ' means there exist indices $k_{1}, \ldots, k_{m-1}$ such that $A_{1}+\cdots+A_{k_{1}}=D_{1}$ and, for $i=2, \ldots, m-1, A_{k_{i-1}+1}+\cdots+A_{k_{i}}=D_{i}$. Taking the $k_{i}$ 's to be minimal, the expression for $Q_{a}\left(w^{*}\right)$ simplifies to

$$
\frac{1}{a^{n}} \sum_{0=k_{0}<\cdots<k_{m-1}<a}\left(a-k_{m-1}\right)^{D_{m}} \prod_{j=1}^{m-1}\left(\left(k_{j}-k_{j-1}\right)^{D_{j}}-\left(k_{j}-k_{j-1}-1\right)^{D_{j}}\right) .
$$

The result now follows from Proposition 4.1

Remarks on Table 2. We calculate SEP after repeated 2-shuffles for various decks using Theorem 4.2. (blackjack) 9 ranks with 4 cards each and another rank with 16 cards; ( $\diamond \diamond \nabla \mathbf{0}) 4$ distinct suits of 13 cards each; (A)) the ace of spades and 51 other cards; (redblack) a two color deck with 26 of either color; and $(0+1) \square$ ) a deck with 5 cards in each of 5 suits. The missing entries in Table 2 highlight the limitations of exact calculations using Theorem 4.2

Remark 4.3 Comparing the data in Table 2 for A and redblack shows that these two cases are remarkably similar. Indeed, both cases exhibit the same asymptotic behavior, which is remarkable since the A has a state space of size 52 while redblack has a state space of size around $5 \times 10^{14}$. 
Tab. 2: Separation distance for $k$ shuffles of 52 cards.

\begin{tabular}{|c|c|c|c|c|c|c|c|c|c|c|c|c|}
\hline $\mathrm{k}$ & 1 & 2 & 3 & 4 & 5 & 6 & 7 & 8 & 9 & 10 & 11 & 12 \\
\hline BD-92 & 1.00 & 1.00 & 1.00 & 1.00 & 1.00 & 1.00 & 1.00 & .995 & .928 & .729 & .478 & .278 \\
\hline blackjack & 1.00 & 1.00 & 1.00 & 1.00 & .999 & .970 & & & & & & \\
\hline $\boldsymbol{p} \triangleright \nabla \phi$ & 1.00 & .997 & .997 & .976 & .884 & .683 & .447 & .260 & .140 & .073 & & \\
\hline A & 1.00 & 1.00 & .993 & .875 & .605 & .353 & .190 & .098 & .050 & .025 & .013 & .006 \\
\hline redblack & .890 & .890 & .849 & .708 & .508 & .317 & .179 & .095 & .049 & .025 & .013 & .006 \\
\hline $0+\mathbb{B} \square$ & 1.00 & 1.00 & .993 & .943 & .778 & .536 & .321 & .177 & & & & \\
\hline
\end{tabular}

Now we derive a basic asymptotic tool which allows asymptotic approximations for general decks.

Proposition 4.4 Let $m \geq 2$ and a be natural numbers, let $\xi_{1}, \ldots, \xi_{m}$ be real numbers in $[0,1]$. Let $r_{1}$, $\ldots, r_{m}$ be natural numbers all at least $r \geq 2$. Let

$$
S_{m}(a ; \underline{\xi}, \underline{r})=\sum_{\substack{a_{1}, \ldots, a_{m} \geq 0 \\ a_{1}+\ldots+a_{m}=a}}\left(a_{1}+\xi_{1}\right)^{r_{1}} \cdots\left(a_{m}+\xi_{m}\right)^{r_{m}} .
$$

Then

$$
\begin{aligned}
& \left|S_{m}(a ; \underline{\xi}, \underline{r})-\frac{r_{1} ! \cdots r_{m} !}{\left(r_{1}+\ldots+r_{m}+m-1\right) !}\left(a+\xi_{1}+\ldots+\xi_{m}\right)^{r_{1}+\ldots+r_{m}+m-1}\right| \\
& \leq r_{1} ! \cdots r_{m} ! \sum_{j=1}^{m-1}\left(\begin{array}{c}
m-1 \\
j
\end{array}\right)\left(\frac{1}{3(r-1)}\right)^{j} \frac{\left(a+\xi_{1}+\ldots+\xi_{m}\right)^{r_{1}+\ldots+r_{m}+m-1-2 j}}{\left(r_{1}+\ldots+r_{m}+m-1-2 j\right) !} .
\end{aligned}
$$

Consider a general deck of $n$ cards with $D_{i}$ cards labelled $i$. We use Proposition 4.4 to find asymptotics for the separation distance given in Theorem 4.2. The following is our 'rule of thumb.'

Theorem 4.5 For a deck of $n$ cards as above, suppose $D_{i} \geq d \geq 3$ for all $1 \leq i \leq m$. Then we have

$$
\operatorname{SEP}(a)=1-(1+\eta) \frac{a^{m-1}}{(n+1) \cdots(n+m-1)} \sum_{j=0}^{m-1}(-1)^{j}\left(\begin{array}{c}
m-1 \\
j
\end{array}\right)\left(1-\frac{j}{a}\right)^{n+m-1},
$$

where $\eta$ is a real number satisfying

$$
|\eta| \leq\left(1+\frac{n^{2}}{3(d-2)(a-m+1)^{2}}\right)^{m-1}-1 .
$$

Proof: To evaluate the expression in Theorem 4.2, we require an understanding of

$$
\sum_{\substack{a_{1}+\ldots+a_{m}=a \\ a_{j} \geq 1}} a_{m}^{D_{m}} \prod_{j=1}^{m-1}\left(a_{j}^{D_{j}}-\left(a_{j}-1\right)^{D_{j}}\right)=\int_{0}^{1} \ldots \int_{0}^{1} \sum_{\substack{a_{1}+\ldots+a_{m}=a \\ a_{j} \geq 1}} a_{m}^{D_{m}} \prod_{j=1}^{m-1}\left(D_{j}\left(a_{j}-1+\xi_{j}\right)^{D_{j}-1} d \xi_{j}\right) .
$$


We now invoke Proposition 4.4 Thus the above equals for some $|\theta| \leq 1$

$$
\begin{aligned}
& \prod_{j=1}^{m} D_{j} ! \int_{0}^{1} \ldots \int_{0}^{1}\left(\frac{\left(a-(m-1)+\xi_{1}+\ldots+\xi_{m-1}\right)^{n}}{n !}+\right. \\
& \left.+\theta \sum_{j=1}^{m-1}\left(\begin{array}{c}
m-1 \\
j
\end{array}\right)\left(\frac{1}{3(d-2)}\right)^{j} \frac{\left(a-(m-1)+\xi_{1}+\ldots+\xi_{m-1}\right)^{n-2 j}}{(n-2 j) !}\right) d \xi_{1} \cdots d \xi_{m-1} .
\end{aligned}
$$

We may simplify the above as

$$
\left(1+\theta\left\{\left(1+\frac{n^{2}}{3(d-2)(a-m+1)^{2}}\right)^{m-1}-1\right\}\right) \frac{D_{1} ! \cdots D_{m} !}{n !} \int_{0}^{1} \cdots \int_{0}^{1}\left(a-m+1+\xi_{1}+\cdots+\xi_{m-1}\right)^{n} d \xi_{1} \cdots d \xi_{m-1}
$$

and evaluating the integrals above this is

$$
\left(1+\theta\left\{\left(1+\frac{n^{2}}{3(d-2)(a-m+1)^{2}}\right)^{m-1}-1\right\}\right) \frac{D_{1} ! \cdots D_{m} !}{n !} \sum_{j=0}^{m-1}(-1)^{j}\left(\begin{array}{c}
m-1 \\
j
\end{array}\right)(a-j)^{n-m+1} .
$$

The Theorem follows.

For simplicity we have restricted ourselves to the case when each pile has at least three cards. With more effort we could extend the analysis to include doubleton piles. The case of some singleton piles

\begin{tabular}{|c|c|c|c|c|c|c|c|c|c|c|c|c|}
\hline $\mathrm{k}$ & 1 & 2 & 3 & 4 & 5 & 6 & 7 & 8 & 9 & 10 & 11 & 12 \\
\hline BD-92 & 1.00 & 1.00 & 1.00 & 1.00 & 1.00 & 1.00 & 1.00 & .995 & .928 & .729 & .478 & .278 \\
\hline blackjack & 1.00 & 1.00 & 1.00 & 1.00 & .999 & .970 & .834 & .596 & .366 & .204 & .108 & .056 \\
\hline $\mathbf{c}\rangle \nabla\rangle$ & 1.00 & 1.00 & .997 & .976 & .884 & .683 & .447 & .260 & .140 & .073 & .037 & .019 \\
\hline redblack & .962 & .925 & .849 & .708 & .508 & .317 & .179 & .095 & .049 & .025 & .013 & .006 \\
\hline O+《 $\square$ & 1.00 & 1.00 & .993 & .943 & .778 & .536 & .321 & .177 & .093 & .048 & .024 & .012 \\
\hline
\end{tabular}
needs some modifications to our formula, but this variant can also be worked out. Below we use our rule of thumb to calculate separation for the same decks as in Table 2 .

Tab. 3: Rule of Thumb for the separation distance for $k$ shuffles of 52 cards.

Remarks on Table 3. The first row gives exact results from the Bayer-Diaconis formula for the full permutation group. The other numbers are from the rule of thumb. Roughly, the single card or red-black numbers suggest that half the usual number of shuffles suffice. The Black-Jack (equivalently Baccarat) numbers suggest a savings of two or three shuffles, and the suit numbers lie in between. The final row is the rule of thumb for the Zener deck with 25 cards, 5 cards for each of 5 suits.

While asymptotic, Theorem 4.5 is astonishingly accurate for decks of practical interest. For instance, comparing exact calculations in Table 2 with approximations using this rule of thumb in Table 3 shows 
that after only 3 shuffles, the numbers agree to the given precision. Moreover, the simplicity of the formula in Theorem 4.5 allows much further computations than are possible using the formula in Theorem 4.2 .

We now give a heuristic for why our rule of thumb is numerically so accurate. For $k \geq 0$, define

$$
f_{k}(z)=\sum_{r=0}^{\infty} r^{k} z^{r}=\frac{A_{k}(z)}{(1-z)^{k+1}}
$$

where $A_{k}(z)$ denotes the $k$-th Eulerian polynomial. The sum over $a_{1}, \ldots, a_{m}$ appearing in our proof of Theorem 4.5 is simply the coefficient of $z^{a}$ in the generating function $(1-z)^{m-1} f_{D_{1}}(z) \cdots f_{D_{m}}(z)$. Our rule of thumb may be interpreted as saying that

$$
(1-z)^{m-1} f_{D_{1}}(z) \cdots f_{D_{m}}(z) \approx \frac{D_{1} ! \cdots D_{m} !}{(n+m-1) !}(1-z)^{m-1} f_{n+m-1}(z) .
$$

To explain the sense in which 9 holds, note that $f_{k}(z)$ extends meromorphically to the complex plane, and it has a pole of order $k+1$ at $z=1$. Moreover it is easy to see that $f_{k}(z)-k ! /(1-z)^{k+1}$ has a pole of order at most $k$ at $z=1$. Therefore, the LHS and RHS of (9) have poles of order $n+1$ at $z=1$, and their leading order contributions match. Therefore the difference between the RHS and LHS of (9) has a pole of order at most $n$ at $z=1$. But in fact, this difference can have a pole of order at most $n-d$ at $z=1$, and thus the approximation in $(9)$ is tighter than what may be expected a priori. To obtain our result on the order of the pole, we record that one can show

$$
f_{k}(z)=\frac{k !}{(1-z)^{k+1}}\left(\frac{(z-1)}{\log z}\right)^{k+1}+\zeta(-k)+O(1-z) .
$$

\section{Gilbreath principle at work}

Conger and Viswanath note that the initial configuration can affect the speed of convergence to stationary. Perhaps this is most striking in the case of Section 3 where a single card is tracked. Recall Table 1 , giving calculations for the distinguished card beginning at the bottom of a deck of 52 cards. In contrast, Table 4 gives calculations for the distinguished card starting in the middle, at position 26. For the latter, both total variation and separation are indistinguishable from zero after only four shuffles.

Tab. 4: Distance to uniformity for a single card starting at the middle of a 52 card deck.

\begin{tabular}{c|rrrr}
\hline & 1 & 2 & 3 & 4 \\
\hline$T V$ & .494 & .152 & .001 & .000 \\
SEP & 1.00 & .487 & .003 & .000 \\
\hline
\end{tabular}

Consider next a deck with $n$ red and $n$ black cards. First take the starting condition of all reds atop all blacks. If the initial cut is at $n$ (the most likely value) then the red-black pattern is perfectly mixed after a single shuffle. More generally, the chance of the deck $w$ resulting from a single 2-shuffle of a deck with $n$ red cards atop $n$ black cards is given by

$$
Q_{2}(w)=\frac{1}{2^{2 n}}\left(2^{\mathrm{h}(w)}+2^{\mathrm{t}(w)}-1\right),
$$


where $\mathrm{h}(w)$ is the number of red cards before the first black card and $\mathrm{t}(w)$ is the number of black cards after the final red card; see [?]. In particular, the total variation after a single 2-shuffle is

$$
\left\|Q_{2}-U\right\|_{T V}=\frac{1}{2}\left(\left(\frac{2^{n+1}-1}{2^{2 n}}-\frac{1}{\left(\begin{array}{c}
2 n \\
n
\end{array}\right)}\right)+\sum_{i=0}^{n-1} \sum_{j=0}^{n-1}\left|\frac{2^{i}+2^{j}-1}{2^{2 n}}-\frac{1}{\left(\begin{array}{c}
2 n \\
n
\end{array}\right)}\right|\left(\begin{array}{c}
2 n-(i+j+2) \\
n-(i+1)
\end{array}\right)\right)
$$

Evaluating this formula for $2 n=52$ give a total variation of 0.579 .

Now take the starting condition to alternate red black red black, etc. As motivation, we recall a popular card trick: Begin with a deck of $2 n$ cards arranged alternately red, black, red, black, etc. The deck may be cut any number of times. Have the deck turned face up and cut (with cuts completed) until one of the cuts results in the two piles having cards of opposite color uppermost. At this point, ask one of the participants to riffle shuffle the two piles together. The resulting arrangement has the top two cards containing one red and one black, the next two cards containing one red and one black, and so on throughout the deck. This trick is called the Gilbreath Principle after its inventor, the mathematician Norman Gilbreath. It is developed, with many variations, in Chapter 4 of [18]. From the trick we see that beginning with an alternating deck severely limits the possibilities. Analyzing the trick reveals the following formula,

$$
2^{2 n} \cdot Q_{2}(w)=\left\{\begin{array}{cl}
2^{n-1}+2^{n} & \text { if } w \text { is the initial alternating deck } \\
2^{n-1} & \text { if } w \text { can result from an odd cut } \\
2^{n} & \text { if } w \text { can result from an even cut } \\
0 & \text { otherwise }
\end{array}\right.
$$

where an odd (resp. even) cut refers to the parity of cards in either pile. From this we compute

$$
\left\|Q_{2}-U\right\|_{T V}=\frac{1}{2}\left(1-\frac{2^{n}+2^{n-1}-1}{\left(\begin{array}{c}
2 n \\
n
\end{array}\right)}\right),
$$

which goes to .5 exponentially fast as $n$ goes to infinity, and indeed is already .500 for $2 n=52$. In contrast, starting with reds above blacks, asymptotic analysis of (10) shows that the total variation tends to 1 after a single shuffle when $n$ is large. Thus again an alternating start leads to faster mixing.

\section{References}

[1] D. Aldous. Random walks on finite groups and rapidly mixing Markov chains. In Seminar on probability, XVII, volume 986 of Lecture Notes in Math., pages 243-297. Springer, Berlin, 1983.

[2] D. Aldous and P. Diaconis. Shuffling cards and stopping times. Amer. Math. Monthly, 93(5):333348, 1986.

[3] D. Bayer and P. Diaconis. Trailing the dovetail shuffle to its lair. Ann. Appl. Probab., 2(2):294-313, 1992.

[4] S. Boyd, P. Diaconis, P. Parrilo, and L. Xiao. Symmetry analysis of reversible Markov chains. Internet Math., 2(1):31-71, 2005. 
[5] T. Ceccherini-Silberstein, F. Scarabotti, and F. Tolli. Harmonic analysis on finite groups, volume 108 of Cambridge Studies in Advanced Mathematics. Cambridge University Press, Cambridge, 2008. Representation theory, Gelfand pairs and Markov chains.

[6] G.-Y. Chen and L. Saloff-Coste. The cutoff phenomenon for randomized riffle shuffles. Random Structures Algorithms, 32(3):346-3745, 2008.

[7] M. Ciucu. No-feedback card guessing for dovetail shuffles. Ann. Appl. Probab., 8(4):1251-1269, 1998.

[8] M. Conger and D. Viswanath. Riffle shuffles of decks with repeated cards. Ann. Probab., 34(2):804819, 2006.

[9] M. Conger and D. Viswanath. Normal approximations for descents and inversions of permutations of multisets. J. Theoret. Probab., 20(2):309-325, 2007.

[10] P. Diaconis. Group representations in probability and statistics. Institute of Mathematical Statistics Lecture Notes-Monograph Series, 11. Institute of Mathematical Statistics, Hayward, CA, 1988.

[11] P. Diaconis. Mathematical developments from the analysis of riffle shuffling. In Groups, combinatorics \& geometry (Durham, 2001), pages 73-97. World Sci. Publ., River Edge, NJ, 2003.

[12] P. Diaconis and J. Fulman. Carries, shuffling and an amazing matrix. preprint, 2008.

[13] P. Diaconis and S. P. Holmes. Random walks on trees and matchings. Electron. J. Probab., 7:no. 6, 17 pp. (electronic), 2002.

[14] P. Diaconis, M. McGrath, and J. Pitman. Riffle shuffles, cycles, and descents. Combinatorica, 15(1):11-29, 1995.

[15] P. Diaconis and M. Shahshahani. Generating a random permutation with random transpositions. $Z$. Wahrsch. Verw. Gebiete, 57(2):159-179, 1981.

[16] A. Fässler and E. Stiefel. Group theoretical methods and their applications. Birkhäuser Boston Inc., Boston, MA, 1992. Translated from the German by Baoswan Dzung Wong.

[17] J. Fulman. Applications of symmetric functions to cycle and increasing subsequence structure after shuffles. J. Algebraic Combin., 16(2):165-194, 2002.

[18] M. Gardner. Martin Gardner's New Mathematical Diversions from Scientific American. Simon \& Schuster, New York, 1966.

[19] E. Gilbert. Theory of shuffling. Technical memorandum, Bell Laboratories, 1955.

[20] J. M. Holte. Carries, combinatorics, and an amazing matrix. Amer. Math. Monthly, 104(2):138-149, 1997.

[21] J. Reeds. Theory of shuffling. Unpublished manuscript, 1976. 
[22] J.-P. Serre. Linear representations of finite groups. Springer-Verlag, New York, 1977. Translated from the second French edition by Leonard L. Scott, Graduate Texts in Mathematics, Vol. 42.

[23] J. R. Weaver. Centrosymmetric (cross-symmetric) matrices, their basic properties, eigenvalues, and eigenvectors. Amer. Math. Monthly, 92(10):711-717, 1985. 
